Andrzej Kompa

Mirosław J. Leszka (Łódź)

\title{
Professor Oktawiusz Jurewicz AS A BYZANTINIST (1926-2016)
}

Professor Oktawiusz Jurewicz, an eminent Polish classicist, philologist and trans-
lator, passed away on 26 April 2016. A doyen of Byzantine studies in Poland, Jurewicz made a significant contribution to their development. Not only did he publish his own works but he also translated works by Byzantine authors, reviewed and edited scholarly texts of his contemporaries, and engaged in organizing academic life. An inspiration to a great number of younger scholars, to whom he was always ready to offer his assistance, Jurewicz belonged to a generation that faced the challenge of organizing scholarly life under very unfavourable conditions - the country suffered international isolation, and those who, because of their experience and significant scholarly achievements, could aspire to the role of the leaders of academic life had either perished during the war or emigrated abroad, never to return. Nevertheless, at the end of his life, he could see his discipline flourish, liberated from previous troubles and limitations. Jurewicz played a significant role in the transformation Polish Byzantinology has undergone over the past few decades. To commemorate his legacy and the lasting influence he had on the students of Byzantium, we wish to present his outstanding scholarly achievements.

While the majority of Polish scholars focused on the study of the Latin and Greek authors of the classical era, Jurewicz turned his linguistic interests to the historiography of the middle Byzantine period. His fundamental accomplishment lay in translating some of the greatest works of Byzantine historiography from the eleventh and twelfth centuries into Polish. The works, which continue to present a number of interpretive challenges, are still vastly used by modern scholars. Aware of how important they were for Byzantine intellectual circles and of the meaning they held outside the Byzantine scholarship, Jurewicz spared no efforts in translating thick volumes of medieval historiography. The first work on which he worked was The Alexiad by Anna Comnena, a daughter of Emperor Alexius I Comnen. Books 1-6 were published in 1969. The publication of the remaining nine books came in $1972^{1}$. An outstanding narrative, due to its literary qualities, its value as

\footnotetext{
${ }^{1}$ Anna Komnena, Aleksjada, vol. I-II, trans. \& comm. O. Jurewicz, Wrocław 1969-1972.
} 
a basic account of the reign of the Comnenian dynasty and the personality of the author, the work attracted the attention of scholars from different fields of study. The Material for History by Nicephorus Bryennios, Anna Comnena's husband, was the next text which Jurewicz set out to translate. Less voluminous than the work by Anna Comnena, but equally important as a source to the history of the empire between 1070-1079, the Material formed something of an introduction to the Alexiad ${ }^{2}$. After translating the Material, he became involved in the translation of the Chronography. Penned by Michael Psellos, a great Byzantine writer and politician ${ }^{3}$, the Chronography is not so much a comprehensive account of political events from 976 to 1077 as a portrayal of Byzantine monarchs from Basil II to Michael VII Ducas. With the author failing to keep his hubris in check and descending into psychologism, the work is hardly an objective exposition of the times about which it tells, yet still of great importance.

Finally, Polish readers could become familiar with the Bibliotheca of Photius. Five volumes, running to more than 1400 pages altogether, were published between 1986 and $1999^{4}$. It was not without some hesitations regarding his ability to complete the ambitious task that Jurewicz decided to become involved in translating these thick volumes. A philological contrariness, to cite his own words, prevailed. However, there were more profound reasons for him to succeed. A humanist and an erudite, deeply fond of all Greek literature, he stressed Photius' significance for preserving the knowledge of the literary output of earlier writers. His own scholarly akríbeia allowed him to achieve the goal of preparing a translation to be used by future generations of Polish scholars. It took a brilliant scholar like Jurewicz to translate so complex a work, and except for the French translation of the text his was the only one that existed at the time.

Jurewicz had the ability to reveal the deep meaning of the works he translated, and it should be kept in mind that, leaving aside Bryennios, he dealt with the most distinguished representatives of Byzantine literature. All the publications mentioned above contained learned introductions yielding new insights into the works and their authors. Despite the passage of time Jurewicz's translations have not become outdated. It remains to be seen whether new critical editions of the Byzantine historiography with which he was concerned will make it necessary to introduce any significant amendments to his work.

In the task of translating works by Byzantine authors, Jurewicz found himself confronted with the issue, which had remained unresolved, of rendering Greek proper names into Polish. The efforts he took in this regard yielded an important publication on the Polonization of Greek names. Although this glossary was

\footnotetext{
${ }^{2}$ Nikefor Bryennios, Materialy historyczne, trans. \& comm. O. Jurewicz, Wrocław 1974.

${ }^{3}$ Micha£ Psellos, Kronika czyli historia jednego stulecia Bizancjum, trans. \& comm. O. Jurewicz, Wrocław 1985.

${ }^{4}$ Focjusz, Biblioteka, vol. I-V, trans. \& comm. O. JuREwicz, Warszawa 1986-1999.
} 
included in the new edition of Georg Ostrogorsky's History of the Byzantine State which was published as late as 2008 , scholars had already been familiar with it, using for some time its typescript version. Although created for practical reasons, it has become an important argument for the protection of the Polish classical onomastics, and even if it is too radical in some of its suggestions, it is more consistent with the Polish language traditions than all the other attempts of this kind and at the same time meets the standards of modern academic and sophisticated Polish.

Jurewicz's long preoccupation with Byzantine historiography resulted in the publication of Historia literatury bizantyńskiej (A History of Byzantine Literature), the only work on the issue that has so far been published in Polish ${ }^{5}$. This concise book, which does not aspire to be a definitive exposition of the subject-matter it deals with, has been used by scholars and students as a convenient general reference on the literature in question. Part of the early Byzantine period is omitted from it as the author starts his analysis with 527. The history of the medieval Greek literature is presented in chronological order and divided into six distinct periods (527-610, 610-717, 717-867, 867-1081, 1081-1204, 1204-1453). Since Jurewicz, following in the tradition of Karl Krumbacher, understood the term 'literature' as applying to all forms of writing, all literary genres are discussed in the History, beginning with historiography and poetry and ending with medicine, mathematics and architecture.

Two other monographs by Jurewicz are the evidence that he was not only an expert on Byzantine literature but was also well versed in a variety of other aspects of the Eastern Roman civilization. One of the monographs mentioned above deals with the person of Emperor Andronicus I Comnenus (1183-1185) ${ }^{6}$. The brief reign of this ruler, usually seen through the prism of bloodshed and its tragic end, received a thorough analysis from Jurewicz who in the account of the emperor's reign managed to modify his image as a cruel tyrant by presenting his rule as one filled with attempts to reform the state machinery (e.g. fiscal system). The book was met with some interest and received reviews from such distinguished scholars as Robert Browning and Alexander Kazhdan ${ }^{7}$. It was subsequently translated into German and published by Hakkert in Amsterdam ${ }^{8}$.

\footnotetext{
${ }^{5}$ Historia literatury bizantyńskiej, Wrocław-Warszawa-Kraków-Gdańsk-Łódź 1984. There were only two works on Byzantine literature published in Polish before 1984: the one by Jan SAJDAK (Literatura bizantyńska, [in:] Wielka literatura powszechna, vol. IV, Warszawa 1933, p. 679-760), and the other by Oktawiusz Jurewicz himself (Literatura bizantyńska, [in:] Dzieje literatur europejskich, ed. W. FloRYAn, Warszawa 1977, p. 137-188).

${ }^{6}$ Andronik I Komnenos, Warszawa 1962.

${ }^{7}$ R. BRowning - EHR 80, 1965, p. 822-823; A. KAŽDAN - VV 24, 1964, p. 253-254; cf. S. WiśNiEWSKI - Bsl 26, 1965, p. 387-389.

${ }^{8}$ Andronikos I. Komnenos, Amsterdam 1970. Rev.: O. Kresten - JÖB 20, 1971, p. 328-334.
} 
The second monograph, Schizma Wschodnia (The eastern schism) ${ }^{9}$, is a popular account of the process resulting in the ultimate split between the Latin and Greek Byzantine Church. Jurewicz introduced the readers to the world of Byzantine civilization, especially to its religious dimension. Another important initiative designed to popularize Byzantine studies concerned the publication of the Byzantine Encyclopedia of culture (Encyklopedia kultury bizantynskiej). The idea of publishing this encyclopedia was born in 1980s, prior to the publication of the Oxford Dictionary of Byzantium or A Biographical Dictionary of the Byzantine Empire. Although the project was finished in 1993, it wasn't until 2002 that the encyclopedia was finally published. 25 scholars were involved in the realization of the project, working under the direction of professor Jurewicz who was the editor of the volume and the author of a few dozens of entries. The encyclopedia remains one of the most ambitious projects of this kind.

In order to fully illuminate the role Jurewicz played in developing Byzantine studies in Poland, it is necessary to mention the efforts he took as an organizer of academic life. He was the first chairman of both the Byzantine Commission of the Committee on Ancient Culture of the Polish Academy of Sciences (KNoKA PAN) and the Polish National Committee of the International Association of the Byzantine Studies (AIEB). Patron of this journal, Professor Waldemar Ceran worked closely with Oktawiusz Jurewicz on the Commission from its inception. Both scholars made a significant contribution to the development of Polish Byzantine studies, inspiring a great number of scholars from around the country. Although Oktawiusz Jurewicz did not create his own school he was the pillar of Polish Byzantinists. He will be remembered as a brilliant scholar and a kind and helpful friend.

\section{Bibliography of works by Oktawiusz Jurewicz (1952-2008) ${ }^{10}$}

\section{2}

Filologia klasyczna $w$ Związu Radzieckim w latach 1945-51, Meander 7.4/5, 1952, p. 226-240.

Nowa organizacja nauczania łaciny $w$ radzieckim szkolnictwie średnim, Meander 7.10, 1952, p. 478-483.

Rec.: P. Grimal, Dictionnaire de la mythologie grecque et romaine, Paris 1951 - Eos 16. 2, 1952, p. 269-270.

\footnotetext{
${ }^{9}$ Schizma wschodnia, Warszawa 1969.

${ }^{10}$ Based on the bibliography placed in M. STARowieyski, Professore Ottavio Jurewicz, [in:] Mélanges d'histoire byzantine offerts à Oktawiusz Jurewicz à l'occasion de Son soixante-dixième anniversaire, ed. W. CERAN, Łódź 1998, p. 6-9; revised and enlarged by the authors.
} 


\section{3}

Szpilka w filologię klasyczna, Problemy 9, 1953, p. 344.

\section{4}

Johannes Franz Stroux, Nauka Polska 2.3, 1954, p. 370-372.

\section{6}

Plautus, Katon Starszy i społeczeństwo rzymskie, Meander 11.12, 1956, p. 447-458.

Konferencja niemieckich i polskich badaczy świata antycznego w Dreźnie, Życie Szkoły Wyższej 1956, 10, p. 72-75.

\section{7}

Z dziejów polskiej bizantynistyki, Meander 12.7/8, 1957, p. 222-240.

Kazimierz Feliks Kumaniecki, 30 lat pracy naukowej i dydaktycznej, Życie Szkoły Wyższej 1957, 12, p. 63-66.

Międzynarodowy kongres poświęcony literaturze nowogreckiej w Berlinie, Życie Szkoły Wyższej $1957,7 / 8$, p. 79-82.

Organizacja i plany naukowe Instytutu Nauk o Grecko-Rzymskiej Kulturze Antycznej przy Niemieckiej Akademii Nauk, Życie Szkoły Wyższej 1957, 10, p. 78-82.

Setna rocznica urodzin G.B. Shawa, Życie Szkoły Wyższej 1957, 1, p. 56-57.

Wstęp, [in:] Arystofanes. Materiały z sesji naukowej Komitetu Nauk o Kulturze Antycznej PAN zorganizowanej na apel Światowej Rady Pokoju w 2400 rocznicę urodzin poety 3-4 grudnia 1954 roku, ed. O. JurewICZ, Wrocław 1957, p. V-VIII.

\section{8}

Niewolnicy w komediach Plauta, Warszawa: Państwowe Wydawnictwo Naukowe 1958, p. 256.

Czy Ksantypa była naprawdę ksantypą, Mówią Wieki 1958, 3, p. 32-33.

Dionysios Solomos (1798-1857), Meander, 1958, p. 265-276 (with J. KuRTIs).

Kazimierz Feliks Kumaniecki (w 30 rocznice pracy naukowej i dydaktyczej), Eos 49.2, 1957/1958, p. 25-44.

Mała encyklopedia świata antycznego, vol. I, Warszawa 1958.

Międzynarodowa konferencja cycerońska, Sprawozdania z Prac Naukowych Wydziału I Nauk Społecznych PAN 1. 4, 1958, p. 63-65.

Neogrecystyka w Polsce, Meander 13.7-8, 1958, p. 81-87.

Rec.: Słownik łacińsko-polski według słownika Hermana Mengego i Henryka Kopii, oprac.

K. Kumaniecki, Warszawa 1957 - Bibliotheca Classica Orientalis, 1958, 3, col. 133-134.

Rec.: K. Kumaniecki, Historia kultury starożytnej Grecji i Rzymu, Warszawa 1955 - Bibliotheca Classica Orientalis, 1958, 4, col. 197-200.

\section{9}

Język łaciński dla lektoratów uniwersyteckich na wydz. filologicznym, historycznym, prawnym, Warszawa: Państwowe Wydawnictwo Naukowe 1959, p. 394 (with L. WinNICZuk, J. ŻuŁaWska; many editions, later as Język łaciński. Podręcznik dla lektoratów szkół wyższych, wyd. 25 - 2004). 
Greckie peregrynacje Juliusza Słowackiego, Meander 14, 1959, p. 177-206.

Die neuegräzistische Forschung in Polen, Berliner Byzantinische Arbeiten 14, 1959, p. 28 -34. Kazimierz Feliks Kumaniecki. Zum 30. Jahrestag seiner wissenschaftlicher und didaktischer

Tätigkeit, Das Altertum 5, 1959, p. 248-256. [= Kazimierz Feliks Kumaniecki, Eos 49.2, 1959 , p. 25-44.]

Plautus, Cato der Ältere und die römische Gesellschaft, [in:] Aus der Altertumskundlichen

Arbeit Volkspolens, hrsg. J. Irmscher, K. Kumaniecki, Berlin 1959, p. 52-72.

System transkrypcyjny alfabetu neogreckiego, [in:] Polskie nazewnictwo geograficzne świata, Warszawa 1959, p. 61-69.

Rec.: A. Krokiewicz, Sokrates, Warszawa 1958 - Kwartalnik Historyczny 66, 1959, p. 219.

Rec.: S. Łoś, Sylwetki rzymskie, Warszawa 1958 - Kwartalnik Historyczny 66, 1959, p. 219.

Rec.: M. Moser-Philtsou, Lehrbuch der neugriechischen Volkssprache, München 1958 - Życie

Szkoły Wyższej 1959, 10, p. 145-148.

\section{0}

Filologia klasyczna na Węgrzech, Meander 15.3, 1960, p. 189-195.

Kabinet pro Studiá Řecká, Řimská a Latinská: Č.A.V. v Praze, Meander 15, 1960, p. 252-254.

Rec.: K. Kumaniecki, Cyceron i jego współcześni, Warszawa 1959, p. 558 - Języki Obce

w Szkole 4. 5, 1960, p. 323-326.

Rec.: Słownik łacińsko-polski, red. M. PleziA, t. I (A-C), Warszawa 1959 - Życie Szkoły Wyższej 1960, 2, p. 124-127.

\section{1}

Bemerkungen über die „servi mali” bei Plautus, Acta Antiqua Academiae Scientiarum Hungaricae $9.1 / 2,1961$, p. 27-36.

Pobyt Andronika Komnenosa na Rusi Halickiej, Sprawozdania z Prac Naukowych Wydziału I Nauk Społecznych PAN 4.5, 1961, p. 48-52.

Rec.: K. Kumaniecki, Cyceron i jego współcześni (Ciceru und seine Zeitgenossen), Warszawa 1959 - Deutsche Literaturzeitung für Kritik der internationalen Wissenschaft 82.3, 1961, p. 225-227.

Rec.: Mała encyklopedia PWN, Warszawa 1959 - Zentralblatt für Bibliothekswesen 75.10, 1961, p. 469-470.

\section{2}

Andronik I Komnenos, Warszawa: Wydawnictwo Naukowe PWN 1962, pp. 176.

* reviews: R. Browning, EHR 80, 1965, p. 822-823; A. KAžDAN, VV 24, 1964, p. 253-254;

S. WIŚNIEWSKI, Bsl 26.2, 1965, p. 387-389.

Komitet Nauk o Kulturze Antycznej PAN, Sprawozdania z Prac Naukowych Wydziału I Nauk Społecznych PAN 5, 1962, p. 142-147.

Mała encyklopedia świata antycznego, vol. II, Warszawa 1962.

Renesans literatury bizantyjskiej w XII w., Meander 17.9, 1962, p. 407-419.

Stefan Srebrny (14 I 1890 - 12 X 1962), Sprawozdania z Prac Naukowych Wydziału I Nauk Społecznych PAN 5.4, 1962, p. 5-6.

Wielka encyklopedia powszechna PWN, vol. I, Warszawa: PWN 1962 (40 entries). 


\section{3}

Miasto o pięciu nazwach (Lygos - Byzantion - Antonia - Konstantinupolis - Istanbul), Meander 18.10, 1963, p. 467-476.

Ze wspomnień ucznia (o prof. St. Srebrnym), Meander 18.2/3, 1963, p. 81-86.

Prof. Dr. Stefan Srebrny, Gelehrter und Übersetzer, Helikon. Rivista di Tradizione e cultura classica dell'Università di Messina 3, 1963, p. 557-558.

Z greckiej literatury nowożytnej, Przegląd Humanistyczny 7.6, 1963, p. 147-150.

\section{4}

Aus der Geschichte der Beziehungen zwischen Byzanz und Rußland in der zweiten Hälfte des 12. Jahrhunderts, [in:] Byzantinische Beiträge, ed. J. IRMSCHER, Berlin 1964, p. 333-357. Kazimierz Kumaniecki (Sylwetki naukowe członków PAN), Nauka Polska 12.1, 1964, p. 68-73. Komisja Bizantynologiczna Komitetu Nauk o Kulturze Antycznej (Rzut oka na polska bizantynistykę), Sprawozdania z Prac Naukowych Wydziału I Nauk Społecznych PAN 7.1, 1964, p. 46-51.

$Z$ recepcji kultury nowogreckiej i klasycznej w Polsce XIX w. Józef Dunin-Borkowski, Eos 54.2, 1964, p. 329-348 [= Przegląd Humanistyczny 9.1, 1965, p. 117-143].

Rec.: Philippi Callimachi Epigrammatum libri duo, ed. C.F. KUMANIECKI, Wratislaviae 1963, pp. 119 - Języki Obce w Szkole 1964, 5.

\section{5}

Rec.: H. Evert-Kappesowa, Studia nad historia wsi bizantyńskiej, Łódź: Łódzkie Towarzystwo Naukowe 1963, pp. 119 - Roczniki Dziejów Społeczno-Gospodarczych 27, 1965, p. $157-158$.

\section{7}

Religia w komediach Plauta, Euhemer. Przegląd Religioznawczy 4/5, 1967, p. 11-18.

Der Vermittlung des antiken Erbes in der Volksrepublik Polen, Das Altertum 13.1, 1967, p. 56-64.

Walki obrazoburcówz ikonodulami w Bizancjum (717-843), Meander 22.6, 1967, p. 269-282.

Edition of K.F. Kumaniecki, Scripta minora, praef. V. Steffen, Academiae scientiarum Polonae Classis prima, Wratislaviae: Zakład Narodowy im. Ossolińskich 1967. Bibliography of Kazimierz Kumaniecki p. XXXI-XLI.

\section{8}

Das römische Theater in Polen im Wandel der Jahrhunderte, [in:] Antiquitas Graeco-Romana ac tempora nostra. Acta Congressus internationalis habiti Brunae diebus 12-16 mensis Aprilis MCMLXVI, ed. J. Burian, L. Vidman, Praguae 1968, p. 295-300.

Über die Religion des Plautus, [in:] Studien zur Geschichte und Philosophie des Altertum, ed. J. Harmatta, Budapest 1968, p. 104-112.

Starożytni Grecy i Rzymianie w życiu prywatnym i państwowym, Warszawa: Państwowe Wydawnictwo Naukowe 1968 (second edition in 1970, third edition 1973; co-author Lidia WinNiCZuK).

Mały słownik pisarzy świata, Warszawa 1968 (31 entries). 


\section{9}

Schizma wschodnia, Warszawa: Książka i Wiedza 1969, pp. 279.

* rec.: R. Ciocan-Ivănescu, B 41, 1971, p. 556-557; A. Ferenc, Euhemer 15, 1971, p. 105-109.

Anna Komnena, Aleksjada, vol. I, trans. et comm. O. Jurewicz, Wrocław: Zakład Narodowy im. Ossolińskich 1969, pp. 184 (second edition in 2005).

Anna Komnena, Aleksjada. Wstęp, trans. O. Jurewicz, Meander 24. 2, 1969, p. 85-92.

$Z$ „Aleksjady” Anny Komneny, Meander 24.2, 1969, p. 83-92.

Rec.: Prokopiusz z Cezarei, Historia sekretna, tłum, wstęp, przypisy A. Konarek, Warszawa: PIW 1969, pp. 224 - Nowe Książki 1969, 16, p. 1108-1109.

\section{0}

Andronikos I. Komnenos, Amsterdam: Hakkert 1970, pp. 159.

* rec.: O. KREsten, JÖB 20, 1971, p. 328-334.

Rec.: H-W. Haußig, Historia kultury bizantyńskiej, tłum. T. Zabłudowski, Warszawa 1969, pp. 548 - Nowe Książki 1970, 7, p. 409-410.

\section{2}

Anna Komnena, Aleksjada, vol. II, trans. et comm. O. Jurewicz, Wrocław: Zakład Narodowy im. Ossolińskich 1972, pp. 298 (second edition in 2005).

Lenin w kręu kultury antycznej, Eos 60, 1972, p. 166-178.

\section{3}

Rec.: S. Runciman, Ostatni renesans bizantyński, tłum. J. Marzęcki, Warszawa 1973, pp. 113 - Nowe Książki 1973, 16, p. 47.

Rec.: Wielki Kościót w niewoli. Studium historyczne patriarchatu konstantynopolitańskiego od czasów bezpośrednio poprzedzających jego podbój przez Turków aż do wybuchu greckiej wojny o niepodległość, tłum. J.S. Łoś, Warszawa 1973, pp. 478 - Argumenty 17 (33), 1973.

\section{4}

Nikefor Bryennios, Materiały historyczne, trans. et comm. O. Jurewicz, Wrocław: Zakład Narodowy im. Ossolińskich 1974, pp. xvi, 98 (second edition in 2006, pp. 205).

L'antiquité classique au cours 25 années de la République Populaire de Pologne, ed. O. JurewICZ, Wrocław: Zakład Narodowy im. Ossolińskich - Wydawnictwo PAN 1974.

Filologia nowogrecka $w$ Polsce, [in:] Bałkanistyka polska. Materiały z posiedzeń naukowych Komisji Bałkanistycznej przy Komitecie Słowianoznawstwa PAN w latach 1972-1973, ed. Z. Stieber, K. Feleszko, J. Reychman, Wrocław: Zakład Narodowy im. Ossolińskich 1974 , p. 55-70.

\section{7}

Literatura bizantyńska, [in:] Dzieje literatur europejskich, ed. W. FlorYAN, Warszawa 1977, p. 137-189. 


\section{4}

Historia literatury bizantyńskiej. Zarys, Wrocław-Warszawa-Kraków-Gdańsk-Łódź: Zakład Narodowy im. Ossolińskich - Wydawnictwo Ossolineum 1984, pp. 354 (second edition in 2007, pp. 320).

"Die Chronographie” des Michael Psellos als Quelle zur byzantinischen Kultur im Ausgang des 10. Jahrhunderts, Eos 72.2, 1984, p. 315-322.

* repr. Byzantinische Forschungen 16, 1991, p. 137-146.

Rec.: H. Cichocka, Przegląd Humanistyczny 29.1/2, 1985, p. 167-171; K. ILsKi, Balcanica Posnaniensia 5, 1990, p. 422.

Kazimierz Feliks Kumaniecki, filolog - tłumacz - nauczyciel - organizator nauki (18 V 1905 - 8 VI 1977), Przegląd Humanistyczny 1984, 1, p. 75-102.

Die Sprache und literarische Kunst von Anna Komnene, [in:] Festschrift Johannes Irmscher, Berlin 1984, p. 85-97.

\section{5}

Anna Komnena - Kronprinzessin und Schriftstellerin, trans. G. JANKE, [in:] Griechenland - Byzanz - Europa. Ein Studienband, ed. J. Herrmann, H. Köpstein, R. Müller, Berlin 1985 (= Berliner Byzantinische Arbeiten, 52), p. 50-60.

Michae Psellos, Kronika czyli historia jednego stulecia Bizancjum, trans. et comm. O. Jurewicz, Wrocław: Zakład Narodowy im. Ossolińskich 1985, pp. xxi, 247 (second edition in 2005, pp. 382).

\section{6}

Focjusz, Biblioteka, vol. I, „Kodeksy” 1-150, trans. et comm. O. Jurewicz, Warszawa: Instytut Wydawniczy Pax 1986, pp. 227.

Kwintus Horacjusz Flakkus, Dzieła wszystkie, vol. I, Ody i epody, ed., trans. et comm. O. JuREwicz, Wrocław: Zakład Narodowy im. Ossolińskich 1986, pp. 445.

\section{8}

Focjusz, Biblioteka, vol. II „Kodeksy” 151-222, trans. et comm. O. Jurewicz, Warszawa: Instytut Wydawniczy Pax 1988, p. 266.

Kwintus Horacjusz Flakkus, Gawędy. Listy. Sztuka poetycka, ed., trans. et comm. O. Jurewicz, Wrocław: Zakład Narodowy im. Ossolińskich 1988, pp. 501.

\section{2}

Gramatyka historyczna języka greckiego. Fonetyka - fleksja, Warszawa: Wydawnictwo Naukowe PWN 1992, pp. 326 (second edition in 1999, pp. 329).

\section{3}

Studi di filologia bizantina condotti in Polonia nel secolo XX (una selezione), [in:] La filologia medievale e umanistica greca e latina nel secolo XX. Atti del Congresso Internazionale, Roma, Consiglio Nazionale delle Ricerche, Università La Sapienza, 11-15 dicembre 1989), ed. E. Follieri, G. Orlandi, M. Regoliosi, A. Armati, A. Luzzi, Roma 1993 [Testi e studi bizantini e neoellenici, 7], vol. II, p. 629-637. 


\section{4}

Focjusz, Biblioteka, vol. III, „Kodeksy” 223-237, trans. et comm. O. JuREwicz, Warszawa: Instytut Wydawniczy Pax 1994, pp. 231.

Słownik pisarzy świata, vol. I, Literatura antyczna: starogrecka, bizantyńska, nowogrecka, rzymska. Literatura afrykańska, ed. O. JuRewicz, S. PiŁAszewicz, Warszawa 1994.

Focjusz, Biblioteka, vol. IV, „Kodeksy” 238-248, trans. et comm. O. Jurewicz, Warszawa: Instytut Wydawniczy Pax 1996, pp. 327.

\section{5}

Griechische Motive in der gegenwärtigen polnischen Literatur, [in:] Europäischer Philhellenismus, Antike Motive in der heutigen europäischen Literatur, Würzburg 1995, p. 189-197.

\section{8}

Byzanz in der polnischen Literatur des 19. und 20. Jahrhunderts, [in:] Byzantinische Stoffe und Motive in der europäischen Literatur des 19. und 20. Jahrhunderts, ed. E. KonstaNTINOU, Frankfurt am Main-Berlin-New York-Paris-Wien 1998 [Philhellenische Studien, 6], p. 137-144.

\section{9}

Focjusz, Biblioteka, vol. V, „Kodeksy” 249-280, trans. et comm. O. Jurewicz, Warszawa: Instytut Wydawniczy Pax 1999, pp. 343.

\section{0}

Kwintus Horacjusz Flakkus, Ody i epody, ed., trans. et comm. O. Jurewicz, Warszawa: Wydawnictwo Naukowe PWN 2000.

Kwintus Horacjusz Flakkus, Gawędy; Listy; Sztuka poetycka, ed., trans. et comm. O. Jurewicz, Warszawa: Wydawnictwo Naukowe PWN 2000.

\section{$2000 / 2001$}

Słownik grecko-polski, ed. O. JuREwICz, vol. I-II, Warszawa: Wydawnictwo Szkolne PWN 2000-2001; published anew in one volume Warszawa: Wydawnictwo Sub Lupa 2015.

\section{$2001 / 2006$}

Several anonymous entries in Wielka encyklopedia PWN, vol. I-XXXI, ed. J. WojNowskI, Warszawa 2001-2006.

Signed entry: vol. IV: Bizantyńskie, cesarstwo. Literatura, p. 135-136.

\section{2}

Encyklopedia kultury bizantyńskiej, ed. O. Jurewicz, Warszawa: Wydawnictwa Uniwersytetu Warszawskiego 2002.

Entries: Abba, p. 1; Abbas, p. 1; Akathistos hymnos, p. 10; Akindynos Grzegorz, p. 10; Akojmeci, p. 11; Aleksander, p. 13; Aleksander $z$ Tralles, p. 14; Alfabet - skarga na marność tego świata, p. 20; Anagnosta Konstantyn, p. 24; Archidiakon, p. 47; Archimandryta, p. 47; Archiprezbiter, p. 47; Arseniusz Autorejan, p. 51-52; Balsamon Teodor, p. 65; Barlaam z Kalabrii, p. 66-67; Barsanufiusz, p. 67; Basileopator, p. 67; Bizantynistyka, bizantynologia, p. 81-82; Bizantyńska literatura, 
p. 83-84; Blemmydes Nikefor, p. 102; Cenobici, p. 110; Chrysoberges Maksym, p. 121; Chrysoloras Demetriusz, p. 121; Chrysorrhoas, p. 121; Chrystodulos Jan z Patmos, p. 121; Chumnos Jerzy, p. 123; Chumnos Nikefor, p. 123; Corpus iuris civilis, p. 127; Diakon, p. 140-141; Domestyk, p. 148; Dukat, p. 152; Duks, p. 152; Dyrrachium, p. 153-154; Edessa, p. 154; Ekthesis Chronike, p. 157-158; Etymologicum magnum, p. 163; Fanarion, p. 172; Files Manuel, p. 174-175; Filozofia Ojca Wina, p. 177; Fizjolog, p. 177; Focjusz, p. 178-179; Geometres Jan Kyriotes, p. 186; Halecki (Chalecki) Oskar, p. 195-196; Hammer Seweryn, p. 196-197; Heirmos, p. 197-198; Historia imperatorum, p. 210; Idiomela, p. 215; Igumen, p. 215; Ikonodule, p. 216; Ikonoklaści, obrazoburcy, p. 216; Jan Italos, p. 231; Jan XIV Kalekas, p. 231; Jan Mauropus, p. 235; Jan Moschos, p. 235; Kabasilas Mikołaj, p. 252; Kabasilas Nil, p. 252; Karmania, p. 257; Kasja, p. 259; Katepano, p. 260-261; Kefalas Konstantyn, p. 261-262; Kiparisjota Jan, p. 263; Kleisura, p. 264; Komisja Bizantynologiczna przy Komitecie Nauk o Kulturze Antycznej Polskiej akademii Nauk, p. 266; Komnena Anna, p. 266; Komnenos i Proklos, p. 268; Kontakion, p. 284; Kumaniecki Kazimierz, p. 295; Laura, p. 301; Libaden Andrzej, p. 310-311; Malakes Eutymiusz, p. 322; Mazarisa podróż do Hadesu, p. 335; Megas duks, p. 336; Meletios, p. 337; Meliteniota Teodor, p. 338; Merarcha, p. 338; Mirologia, p. 353; Msza gołobrodych, p. 362; Myriokefalon, p. 363; Nazjanz, p. 365; Nowacjanie, p. 378; Ochryda, p. 378; Ogień grecki, p. 378; Opowieść o czworonogach, p. 381; Pachomiusz, p. 385; Palamas Grzegorz, p. 386; Patrycjuszka zoste, p. 397; Pizydes Jerzy, p. 404; Porikolog, p. 406-407; Psarolog, p. 416; Pulolog, p. 418; Roman Melodos, p. 424; Sajdak Jan, p. 427-428; Scholia, p. 431; Sebastokrator, p. 432; Sebastos, p. 432; Sinko Tadeusz, p. 435-436; Spaneas, p. 441; Spatariusz, p. 441; Sternbach Leon, p. 445; Stratopedarcha, p. 447; Stratores, p. 447; Stylici, p. 447; Synaksarion, p. 449; Syntomon, p. 450; Tagmata, p. 453; Teodor Studyta, p. 459; Teodor z Mopsuestii, p. 459; Tornikes Dymitr, p. 476; Tornikes Grzegorz, p. 476; Troparion, p. 481; Trzy rozdziały, p. 483; Turyn Aleksander, p. 483; Typikon, p. 485; Tzetzes Isaak, p. 486; Ulpian, p. 487; Witkowski Stanisław, p. 493-494; Zakład Filologii Greckiej, Bizantyńskiej i Nowogreckiej Instytutu Filologii Klasycznej Uniwersytetu Warszawskiego, p. 496; Zotyk Paraspondyl, p. 502; Zwierciadło kobiet, p; 502-503; Zygaben (Zygaden) Jan, p. 503.

\section{8}

Zasady spolszczania i transpozycji imion własnych i nazw geograficznych epoki bizantyńskiej, [in:] G. Ostrogorski, Dzieje Bizancjum, trans. W. Ceran, H. Evert-Kappesowa, S. WiśnIEWsKI, ${ }^{2}$ Warszawa 2008, p. 599-626.

\section{Bibliography}

Anna Komnena, Aleksjada, vol. I-II, trans. \& comm. O. Jurewicz, Wrocław 1969-1972.

Focjusz, Biblioteka, vol. I-V, trans. \& comm. O. JuRewicz, Warszawa 1986-1999.

Micha£ Psellos, Kronika czyli historia jednego stulecia Bizancjum, trans. \& comm. O. Jurewicz, Wrocław 1985.

Nikefor Bryennios, Materialy historyczne, trans. \& comm. O. Jurewicz, Wrocław 1974.

Jurewicz O., Andronik I Komnenos, Warszawa 1962.

Jurewicz O., Andronikos I. Komnenos, Amsterdam 1970. Rev.: O. Kresten - JÖB 20, 1971, p. 328-334.

Jurewicz O., Historia literatury bizantyńskiej, Wrocław-Warszawa-Kraków-Gdańsk-Łódź 1984.

Jurewicz O., Literatura bizantyńska, [in:] Dzieje literatur europejskich, ed. W. Floryan, Warszawa 1977, p. 137-188.

Jurewicz O., Schizma wschodnia, Warszawa 1969. 
SAJDAK J., Literatura bizantyńska, [in:] Wielka literatura powszechna, vol. IV, Warszawa 1933, p. 679-760.

Starowieyski M., Professore Ottavio Jurewicz, [in:] Mélanges d’histoire byzantine offerts à Oktawiusz Jurewicz à l'occasion de Son soixante-dixième anniversaire, red. W. CERAN, Łódź 1998, p. 6-9.

Abstract. The authors summarize the academic legacy of late Oktawiusz Jurewicz and his role as a leading Polish byzantinist of the second half of the $20^{\text {th }}$ century. The text is supplemented by the detailed and updated bibliography of Jurewicz.

Keywords: Oktawiusz Jurewicz, classical Philology, Byzantine studies in Poland, history of humanities.

Andrzej Kompa

Uniwersytet Łódzki

Wydział Filozoficzno-Historyczny

Instytut Historii

Katedra Historii Bizancjum

ul. Kamińskiego 27a

90-219 Łódź, Polska/Poland

andrzejkompa@uni.lodz.pl

Mirosław J. Leszka

Uniwersytet Łódzki

Wydział Filozoficzno-Historyczny

Instytut Historii

Katedra Historii Bizancjum

ul. Kamińskiego 27a

90-219 Łódź, Polska/Poland mirleszka@poczta.onet.pl 